\title{
ANTECEDENTES DEL ESTUDIO EN ESTUDIANTES UNIVERSITARIOS: VALIDACION DE UN MODELO DE ECUACIONES ESTRUCTURALES BASADO EN LA TEORÍA DEL COMPORTAMIENTO INTERPERSONAL EN CHILE
}

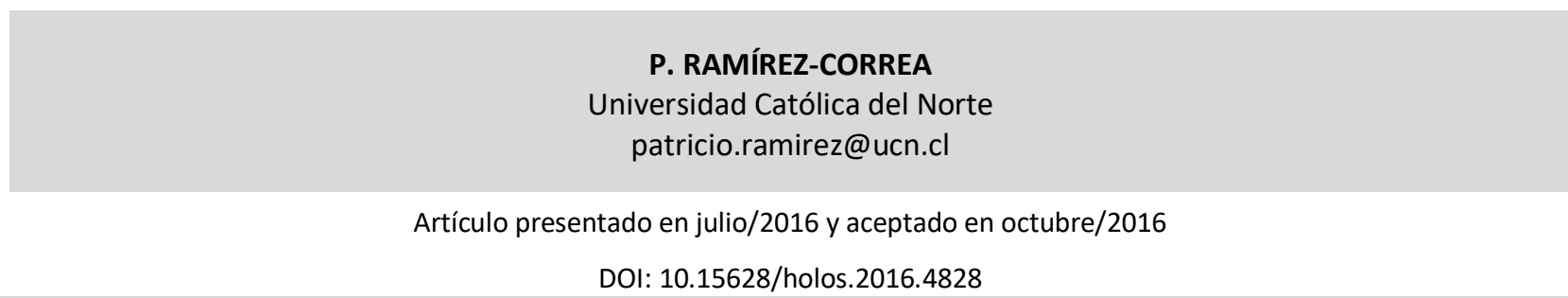

\section{RESUMEN}

El objetivo de este trabajo es explorar los factores que predicen el comportamiento de estudio en los alumnos

fenómeno. El Modelado de Ecuaciones Estructurales fue usado para analizar el modelo. Los resultados indican que universitarios. Se utilizó una encuesta basada en la Teoría del Comportamiento Interpersonal realizada a 137 el modelo explica el $54 \%$ del comportamiento de estudio, estudiantes universitarios chilenos para medir este y apuntan a el hábito de como el antecedente clave de este comportamiento.

PALABRAS CLAVE: Teoría del Comportamiento Interpersonal, Educación Superior, Modelamiento de Ecuaciones Estructurales, Chile.

\section{ANTECEDENTS OF UNDERGRADUATE STUDENTS' STUDY: VALIDATION OF A STRUCTURAL EQUATION MODEL BASED ON THE THEORY OF INTERPERSONAL BEHAVIOUR IN CHILE}

\begin{abstract}
The aim of this paper is to explore the factors that predict the behaviour of study by university students. A survey based on the Theory of Interpersonal Behaviour conducted to 137 Chilean university students was used to measure this phenomenon. Structural Equation
\end{abstract}

Modelling was used to analyse the model. The results indicate that the model explains $54 \%$ of the study behaviour, and point to the habit as the key antecedent of this behaviour.

KEYWORDS: Theory of Interpersonal Behaviour, Higher Education, Structural Equation Modelling, Chile. 


\section{INTRODUCCION}

La motivación es un factor decisivo para el aprendizaje y el rendimiento académico (Tous \& Medinas, 2007). Este hecho es particularmente relevante en la educación superior. En la universidad, y por encima de las habilidades intelectuales de los alumnos, algunos autores indican al esfuerzo como el mayor predictor del éxito académico (Kappe \& Van der Flier, 2012; Kennedy, 2013).

En particular y en el ámbito de los países latinoamericanos, el progreso en cobertura de los sistemas de educación superior ha implicado la creciente incorporación de estudiantes procedentes de niveles socioeconómicos diversos (Donoso \& Schiefelbein, 2007). Esta realidad ha puesto un nuevo desafío a las universidades latinoamericanas: los aumentos en cobertura traen consigo bajas tasas de permanencia y de retención (Fernández-Hileman, Corengia, \& Durand, 2014). Y si bien, las instituciones de educación superior y los gobiernos latinoamericanos han realizado acciones para aumentar estos índices de éxito académico, los resultados de estas propuestas no han sido suficientes (Ortiz, Pérez, \& Salazar, 2015).

Considerando estos antecedentes, esta investigación desea profundizar sobre los motivadores del esfuerzo que realiza el estudiante universitario chileno en su quehacer, pues a la luz de este escrutinio entendemos que es posible que las instituciones de educación superior diseñen planes efectivos para aumentar estos esfuerzos individuales, y por tanto, mejorar la probabilidad de éxito académico de sus estudiantes.

En específico, el objetivo de este trabajo es explorar los factores que predicen la acción de estudiar por parte del alumno universitario. Con un enfoque conductual, se utiliza a la Teoría del Comportamiento Interpersonal (TCI:Triandis, 1980; Triandis, 1977), como marco conceptual para explicar el estudio en universitarios. En el ámbito del individuo, la $\mathrm{TCl}$ explicita factores personales que originan la intención o motivación de realizar una conducta específica, esta intención más el hábito de realizar dicho comportamiento explican la conducta determinada, en función de la condiciones facilitadoras de la acción.

En restante texto del artículo se ha estructurado como sigue. Primero se desarrolla una revisión bibliográfica de la $\mathrm{TCl}$, y a partir de ello se propone un modelo de investigación. A continuación, se explica la metodología de estudio, basada en una encuesta a estudiantes universitarios chilenos y su análisis a través del modelamiento de ecuaciones estructurales a través del método de mínimos cuadrados parciales. Seguido, se presentan los resultados y se realiza la discusión de ellos. Y finalmente, se entrega la conclusión de estudio.

\section{REVISIÓN BIBLIOGRÁFICA}

\subsection{Teoría del Comportamiento Interpersonal}

La $\mathrm{TCl}$ tiene por propósito el estudio del comportamiento individual. En específico, y enraizada en la escuela conductual, la TCl se orienta a entender y explicar a través de factores personales la intención individual de realizar una conducta específica, y como esta conducta es una función de la intención y el hábito de realizar dicha conducta, dado un contexto de condiciones facilitadoras para determinado comportamiento. 
La TCI fue propuesta por Harry Triandis en 1977 (Triandis, 1977), y fue desarrollada en 1980 (Triandis, 1980). En esta teoría los antecedentes de un comportamiento pueden ser descritos como se muestra en la Figura 1.

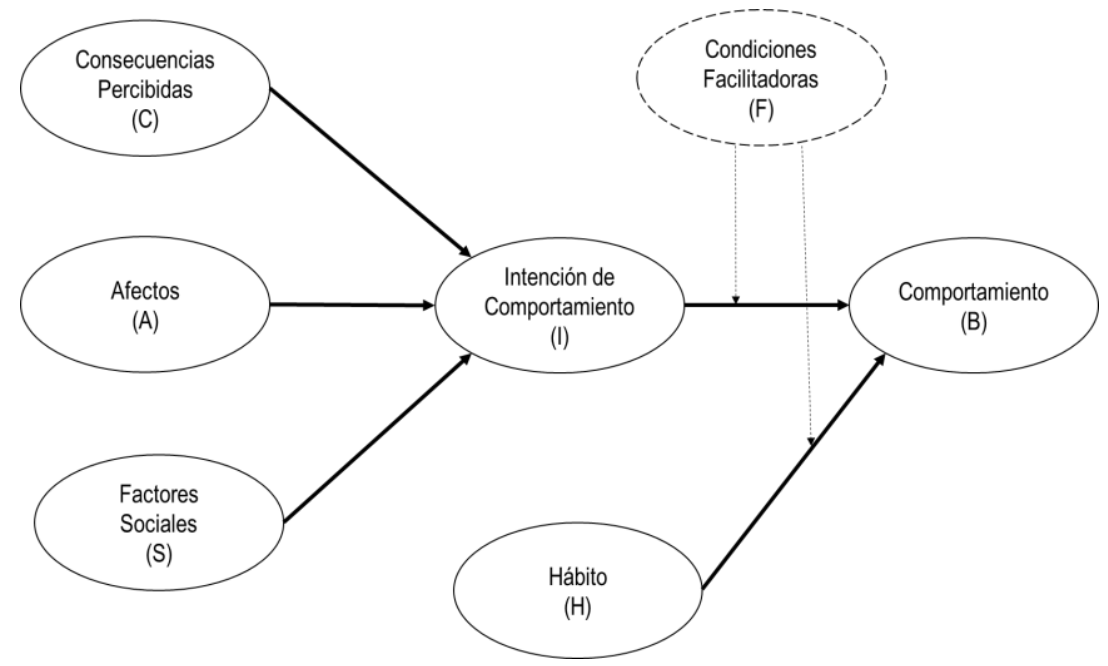

Figura 1: Teoría del Comportamiento Interpersonal. Fuente: Elaboración propia

Primero, el comportamiento es explicado por la intención (I) de realizar este comportamiento y el hábito $(H)$ de su realización. $H$ hace referencia a la disposición de repetir un comportamiento pasado. Segundo, I es una función de los factores sociales (S), los afectos (A) y las consecuencias percibidas (C). S se refiere a la internalización subjetiva de la cultura del grupo de personas con las cuales el individuo interactúa con mayor frecuencia, A se refiere a la respuesta emocional a una situación particular, que se basa en los instintos y procesos inconscientes de la mente, y $\mathrm{C}$ se refiere al valor que el comportamiento puede entregar al individuo después de que él o ella realicen el comportamiento. Tercero, la fuerzas aplicativas de I y $\mathrm{H}$ son moderadas por el grado de condiciones facilitadoras (F) del comportamiento. F se refiere a las limitaciones situacionales que puedan impedir que el individuo realice el comportamiento deseado.

\subsection{Aplicación de la Teoría del Comportamiento Interpersonal}

Desde su introducción la $\mathrm{TCl}$ ha sido adoptada en diversos estudios, en ámbitos y contextos diferentes.

En el ámbito de salud se pueden señalar dos estudios claves. Brown, Baumann, Helberg, Han, Fontana y Love (1996) incluyeron variables de la TCI para predecir la realización de el examen de mamografía anual por parte de pacientes y médicos. Por su parte, Bealanger, Godin, Alaryby Bernard (2002), utilizando un modelo basado en la TCl, llegaron a explicar el uso de preservativos en una muestra de usuarios de drogas inyectables.

En el ambito de las tecnologías de información existen varios ejemplos de su aplicación en distintos contextos. Al-Khaldi y Wallace (1999) utilizaron la TCl para entender la utilización de computadores personales en contexto laboral, sus resultados apoyan la idea que las actitudes individuales, los factores sociales y las condiciones facilitadoras son antecedentes del uso de los computadores en el trabajo. Por su parte, Karahanna y Straub (1999) investigaron el origen de las percepciones de uso y facilidad de uso del correo electrónico con base a la $\mathrm{TCl}$, en una muestra de 100 usuarios, uno de sus principales resultados reveló que las condiciones facilitadoras no tienen 
efecto en la facilidad de uso de esta tecnología. Cheung, Chang y Lai (2000) aplicaron la TCI para predecir el uso de Internet por parte de trabajadores en un contexto empresarial, sus hallazgos indican que los factores sociales y las condiciones facilitadoras son los elementos más importantes para explicar el uso de Internet dentro de la empresa. Por su parte, Limayem y Hirt (2003) estudiaron en un contexto universitario la aceptación de una aplicación computacional de apoyo al aprendizaje basados en la $\mathrm{TCl}$, sus resultados destacan la importancia de considerar los factores conscientes (intención) y subconscientes (hábito) para explicar el comportamiento del uso de la aplicación. Finalmente, y en un contexto laboral, Moody y Siponen (2013) probaron un modelo basado en la $\mathrm{TCl}$ que explica el uso de Internet para propósitos no asociados al trabajo.

En el ámbito del comportamiento ciudadano es posible destacar tres estudios que utilizan la TCl. Zalesny (1985) basado en la TCl comparo exitosamente los factores económicos y no económicos que predicen la preferencia de voto en un representante universitario. Por su parte, Lanken, Aarts, Knippenberg y Knippenberg (1994) compararon la actitud de un ciudadano en relación a su hábito al momento de seleccionar un tipo de vehículo para movilizarse, basados en la $\mathrm{TCl}$ sus resultados indican que tanto actitud como hábito predicen la selección, y además, a medida que la fuerza del hábito aumenta, la actitud pierde fuerza, y viceversa. Finalmente, Bamberg y Schmidt (2003) estudiaron el uso del automóvil para ir a la universidad, en su análisis aplicaron la $\mathrm{TCl}$ y el resultado indica que el hábito es el elemento de mayor relevancia para predecir esta conducta.

En el contexto de estudiantes universitarios existen algunos ejemplos asociados al ámbito de sus comportamientos sexuales. Boyd y Wandersman (1991) concluyen que la TCl tiene un alto poder explicativo del uso de preservativos en estudiantes universitarios. Maticka-Tyndale, Herold y Mewhinney (1998) utilizan la $\mathrm{TCl}$ para explicar el sexo casual de los estudiantes universitarios canadienses en periodos vacacionales, sus resultados indican que los factores sociales y las actitudes personales explican en forma significativa la participación en dichas de actividades. Posteriormente, los primeros dos autores de ese estudio más Oppermann, aplican la TCl para estudiar el sexo casual entre estudiantes australianos, entre sus resultados se destaca que los factores situacionales, la intención y la experiencia sexual previa influyen en establecer este tipo de relaciones (Maticka-Tyndale, Herold, \& Oppermann, 2003).

\section{MODELO DE INVESTIGACIÓN}

Basado en la TCl (Triandis, 1980), en la Figura 2 se presenta el modelo de investigación y las hipótesis propuestas en este estudio. 


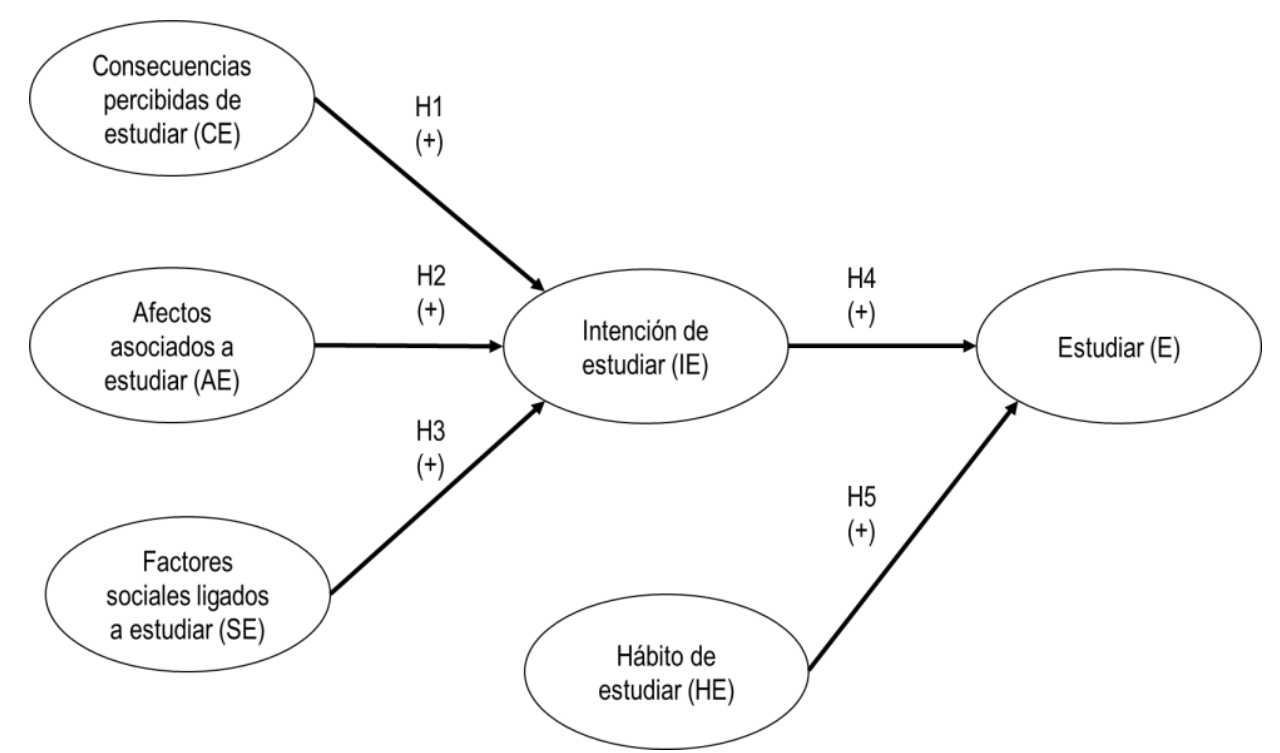

Figura 2: Modelo de investigación e hipótesis.

Fuente: Elaboración propia

En un contexto de alumnos universitarios, el modelo propone que las consecuencias percibidas, los afectos y los factores sociales ligados a estudiar son antecedentes de la intención de estudiar, y además, la acción de estudiar es una función de la intención y el hábito de estudiar.

Autores que han aplicado la $\mathrm{TCl}$ apoyan que la intención de un comportamiento es explicada por las consecuencias percibidas (Moody \& Siponen, 2013; Triandis, 1980), los afectos asociados (Moody \& Siponen, 2013; Triandis, 1980), y los factores sociales ligados al comportamiento (Al-Khaldi \& Wallace, 1999; Maticka-Tyndale et al., 1998; Moody \& Siponen, 2013; Triandis, 1980). Considerando los previos estudios se proponen las siguientes hipótesis:

H1: Las consecuencias percibidas de estudiar están asociadas positivamente a la intención de estudiar en un contexto de educación superior.

H2: Los afectos asociados a estudiar están asociados positivamente a la intención de estudiar en un contexto de educación superior.

H3: Los factores sociales ligados a estudiar están asociados positivamente a la intención de estudiar en un contexto de educación superior.

La literatura ampliamente ha apoyado la idea que la intención de comportamiento es un fuerte predictor del comportamiento (Ajzen, 1991; Ajzen \& Fishbein, 1980; Maticka-Tyndale et al., 2003). En particular la TCI plantea esta relación (Triandis, 1980). Considerando los previos estudios se propone la siguiente hipótesis:

H4: La intención de estudiar está asociada positivamente al comportamiento de estudiar en un contexto de educación superior.

Finalmente, diversos estudios indican que cuando los comportamientos se vuelven habituales con el tiempo, estos comportamientos pueden ser provocados sin la mediación de las intenciones (Bamberg \& Schmidt, 2003; Danner, Aarts, \& Vries, 2008; Lanken et al., 1994; Ouellette \& Wood, 1998; Triandis, 1980). De hecho, el hábito predice el comportamiento futuro más allá de 
las intenciones conscientes (Ouellette \& Wood, 1998). Considerando los previos estudios se propone la siguiente hipótesis:

H5: El hábito de estudiar está asociado positivamente al comportamiento de estudiar en un contexto de educación superior.

\section{METOdOLOGÍA}

\subsection{Muestra}

Los datos para el estudio fueron obtenidos a través de una encuesta realizada cara a cara a estudiantes de pregrado de una universidad chilena. Se utilizó para seleccionar a los encuestados un método de muestreo no aleatorio (muestra de conveniencia). Antes de realizar la encuesta, se realizó un estudio piloto con el propósito de validar el instrumento.

El tamaño total de la muestra es de 137 estudiantes. El 50,4\% de los entrevistados son hombres (69) y el 40,6\% son mujeres (68). La edad promedio es de 22,3 años, con una desviación estándar de 2,15. En cuanto al nivel de avance de los estudiantes encuestados, el progreso académico de la muestra va desde el primer año al último año de sus programas de estudio, y su rendimiento académico promedio es de 4,36, con una desviación estándar de 0,93 (en una escala de 1 a 7 , donde 4 es la nota mínima de aprobación de una asignatura).

\subsection{Escala de medida}

Este estudio utiliza escalas de medición probadas en la literatura. En particular, las escalas para las variables del modelo se adaptaron del estudio de Moody y Siponen (2013), y todos sus ítems se midieron con una escala Likert de cinco puntos (ver Tabla 1).

Tabla 1: Instrumento de medida.

\begin{tabular}{c|ll}
\hline Variable & \multicolumn{1}{c}{ Ítems } \\
\hline $\begin{array}{c}\text { Consecuencias } \\
\text { percibidas de estudiar } \\
\text { (CE) }\end{array}$ & CE1 & Me gusta la idea de estudiar. \\
& CE3 & Estudiar es una buena idea. \\
Estudiar es una idea inteligente. \\
\hline $\begin{array}{c}\text { Afectos asociados a } \\
\text { estudia (AE) }\end{array}$ & AE1 & Siento que estudiar es placentero. \\
& AE3 & Siento que estudiar es interesante. \\
Factores sociales & SE1 & Mi familia aprueba que yo estudie. \\
ligados a estudiar(SE) & SE2 & Mis amigos aprueban que yo estudie. \\
& SE3 & Mis compañeros de universidad aprueban que yo estudie. \\
\hline \multirow{2}{*}{$\begin{array}{c}\text { Intención de estudiar } \\
\text { (IE) }\end{array}$} & IE1 & Intentaré estudiar en el futuro. \\
& IE2 & Voy a estudiar en el futuro. \\
IE3 & Espero estudiar en el futuro. \\
\hline \multirow{2}{*}{ Hábito de estudiar } & HE1 & Estudio frecuentemente. \\
(HE) & HE2 & Comienzo a estudiar de forma automática. \\
& HE3 & Comienzo a estudiar sin tener un recordatorio consciente. \\
& HE4 & Me siento raro si no estudio. \\
\hline \multirow{2}{*}{ Estudiar (E) } & E1 & En general, yo estudio. \\
& E2 & Yo estudio varias veces en la semana. \\
& E3 & Yo gasto una cantidad significativa de tiempo estudiando.
\end{tabular}




\subsection{Herramientas estadísticas}

Para analizar el modelo de investigación propuesto se ha utilizado el modelamiento de ecuaciones estructurales a través del enfoque de mínimos cuadrados parciales (PLS-Partial Least Squares). PLS analiza tanto el modelo de medida como el modelo estructural (Chin, 1998; Tenenhaus, Vinzi, Chatelin, \& Lauro, 2005). En específico, para llevar a cabo estos análisis se aplica el software WarpPLS 5.0 (Kock, 2015).

\section{RESULTADOS Y DISCUSIONES}

El enfoque PLS está definido por dos modelos: el modelo de medida y el modelo estructural. Anterior a poder realizar el análisis del modelo de estructural es necesario estudiar la confiabilidad y validez del modelo de medida. La Tabla 2 muestra las cargas y cargas cruzadas de los indicadores de las variables latentes del modelo, como todos estos valores son mayores a 0,7 , se acepta la fiabilidad individual del modelo de medida.

Tabla 2: Cargas y cargas cruzadas de las variables latentes.

\begin{tabular}{c|c|c|c|c|c|c}
\hline Ítems & $\mathrm{CE}$ & $\mathrm{AE}$ & $\mathrm{SE}$ & $\mathrm{HE}$ & $\mathrm{IE}$ & $\mathrm{E}$ \\
\hline CE1 & $\mathbf{0 , 8 3}$ & 0,15 & $-0,16$ & 0,07 & $-0,07$ & 0,05 \\
CE2 & $\mathbf{0 , 9 2}$ & $-0,03$ & $-0,03$ & 0,00 & 0,08 & $-0,13$ \\
CE3 & $\mathbf{0 , 8 4}$ & $-0,12$ & 0,19 & $-0,07$ & $-0,01$ & 0,09 \\
AE1 & $\mathbf{0 , 1 9}$ & $\mathbf{0 , 8 0}$ & $-0,05$ & 0,03 & $-0,24$ & 0,08 \\
AE2 & $-0,11$ & $\mathbf{0 , 9 2}$ & 0,06 & $-0,02$ & 0,09 & 0,03 \\
AE3 & $-0,06$ & $\mathbf{0 , 8 8}$ & $-0,02$ & $-0,01$ & 0,13 & $-0,11$ \\
SE1 & $-0,18$ & 0,15 & $\mathbf{0 , 7 7}$ & $-0,02$ & 0,04 & 0,03 \\
SE2 & 0,10 & $-0,09$ & $\mathbf{0 , 9 5}$ & 0,03 & 0,00 & $-0,06$ \\
SE3 & 0,05 & $-0,03$ & $\mathbf{0 , 9 2}$ & $-0,01$ & $-0,03$ & 0,04 \\
HE1 & 0,13 & 0,16 & 0,02 & $\mathbf{0 , 8 3}$ & $-0,18$ & 0,17 \\
HE2 & 0,01 & $-0,07$ & $-0,04$ & $\mathbf{0 , 9 1}$ & 0,01 & $-0,02$ \\
HE3 & $-0,18$ & 0,05 & $-0,06$ & $\mathbf{0 , 8 1}$ & 0,15 & $-0,34$ \\
HE4 & 0,03 & $-0,13$ & 0,09 & $\mathbf{0 , 8 0}$ & 0,03 & 0,19 \\
IE1 & $-0,06$ & 0,25 & $-0,04$ & $-0,01$ & $\mathbf{0 , 9 1}$ & $-0,16$ \\
IE2 & 0,07 & $-0,11$ & $-0,04$ & 0,05 & $\mathbf{0 , 9 3}$ & 0,01 \\
IE3 & $-0,01$ & $-0,15$ & 0,08 & $-0,04$ & $\mathbf{0 , 8 6}$ & 0,16 \\
E1 & $-0,06$ & 0,14 & 0,03 & 0,16 & 0,18 & $\mathbf{0 , 8 3}$ \\
E2 & 0,10 & $-0,06$ & 0,01 & $-0,10$ & 0,02 & $\mathbf{0 , 9 2}$ \\
E3 & $-0,05$ & $-0,07$ & $-0,04$ & $-0,05$ & $-0,20$ & $\mathbf{0 , 8 5}$ \\
\hline
\end{tabular}

En la Tabla 3 se muestran los coeficientes Confiabilidad Compuesta (CC), Alfa de Cronbach (AC) y Varianza Media Extraída (Average Variance Extracted -AVE). Dado que los valores de CC y AC son superiores a 0,8 para todas las variables, se acepta la fiabilidad de los constructos del modelo de medida. Adicionalmente, considerando que el valor de AVE es mayor que 0,5 para todas las variables, se acepta su validez convergente. 
Tabla 3: Coeficientes de las variables latentes.

\begin{tabular}{c|c|c|c}
\hline $\begin{array}{c}\text { Variable } \\
\text { Latente }\end{array}$ & $\begin{array}{c}\text { Confiabilidad } \\
\text { Compuesta }\end{array}$ & $\begin{array}{c}\text { Alfa de } \\
\text { Cronbach }\end{array}$ & AVE \\
\hline CE & 0,90 & 0,82 & 0,74 \\
AE & 0,90 & 0,83 & 0,75 \\
SE & 0,92 & 0,86 & 0,78 \\
HE & 0,90 & 0,86 & 0,70 \\
IE & 0,93 & 0,88 & 0,81 \\
E & 0,90 & 0,83 & 0,75 \\
\hline
\end{tabular}

La Tabla 4 muestra la prueba de la validez discriminante del modelo de medida, en ella se comprueba la superioridad numérica de la raíz cuadrada de la AVE de cada constructo, los valores en la diagonal, sobre las correlaciones entre los constructos, los otros valores (Fornell \& Larcker, 1981).

Tabla 4: Validez discriminante de las variables latentes.

\begin{tabular}{c|c|c|c|c|c|c}
\hline $\begin{array}{c}\text { Variable } \\
\text { Latente }\end{array}$ & $\mathrm{CE}$ & $\mathrm{AE}$ & $\mathrm{SE}$ & $\mathrm{HE}$ & $\mathrm{IE}$ & $\mathrm{E}$ \\
\hline $\mathrm{CE}$ & $\mathbf{0 , 8 6}$ & & & & & \\
$\mathrm{AE}$ & 0,57 & $\mathbf{0 , 8 7}$ & & & & \\
$\mathrm{SE}$ & 0,28 & $\mathbf{0 , 2 6}$ & $\mathbf{0 , 8 9}$ & & & \\
$\mathrm{HE}$ & 0,44 & 0,48 & 0,24 & $\mathbf{0 , 8 4}$ & & \\
$\mathrm{IE}$ & 0,38 & $\mathbf{0 , 4 6}$ & 0,29 & 0,25 & $\mathbf{0 , 9 0}$ & \\
$\mathrm{E}$ & 0,36 & 0,50 & 0,26 & 0,67 & 0,41 & $\mathbf{0 , 8 7}$ \\
\hline
\end{tabular}

Luego del análisis del modelo de medida se realizó el análisis del modelo estructural. La Tabla 5 muestra los índices globales de ajuste del modelo, el valor de bondad de ajuste de Tenenhaus (GoF) de 0,56 comprueba la confiabilidad y el ajuste del modelo (Tenenhaus et al., 2005).

Tabla 5: Índices de ajuste del modelo.

\begin{tabular}{l|l}
\hline Promedio de los coeficientes de camino & 0,31 \\
Promedio de los R cuadrado & 0,41 \\
Bondad de ajuste (GoF) & 0,56 \\
\hline
\end{tabular}

La Figura 3 muestra los betas calculados en el análisis del modelo estructural. Las hipótesis fueron verificadas a través de la exploración de los coeficientes de camino. Un proceso de remuestreo utilizando el método Stable3 (Kock, 2015) se utilizó para analizar la significación estadística de cada camino. En base a estos valores se aceptan las hipótesis H1, H2, H3, H4 y H5, ya que las relaciones propuestas son estadísticamente significativas. 


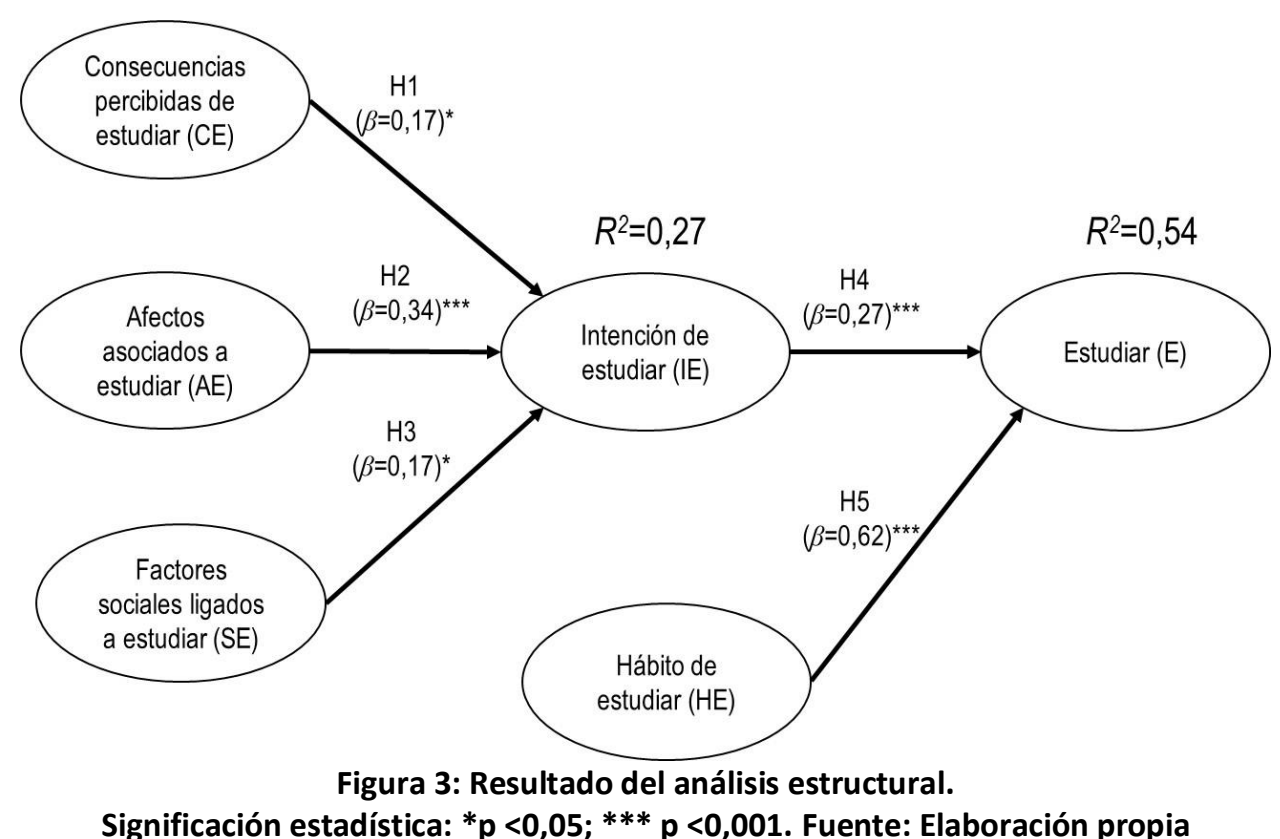

En forma global, este análisis valida la aplicabilidad de la $\mathrm{TCl}$ para explicar el estudio en estudiantes universitarios. Esta aplicabilidad se soporta en dos elementos. Primero, en la validez y fiabilidad del modelo de medida, donde se destacan los valores alcanzados por los coeficiente Alfa de Cronbach y AVE para cada variable del modelo. Y segundo, en la alta calidad del ajuste global del modelo propuesto $(\mathrm{GoF}=0,56)$, evaluación de calidad que considera tanto el modelo de medida como el modelo estructural.

En particular y en relación al análisis del modelo estructural, los resultados señalan la importancia del hábito de estudiar para explicar el estudio, de hecho y comparado con la intención, $\mathrm{HE}(\beta=0,62)$ surge como el predictor más significativo, duplicando ampliamente la importancia de la IE $(\beta=0,27)$. Este hallazgo es consistente con la aplicación de la TCl en otros ámbitos (Bamberg \& Schmidt, 2003; Moody \& Siponen, 2013), y apoya la idea que a nivel individual el hábito predice el comportamiento futuro más allá de las intenciones conscientes (Ouellette \& Wood, 1998). Por otra parte, de los tres antecedentes que explican la intención de estudiar en el modelo, los afectos asociados a este comportamiento aparecen como el factor más importante, en concreto, la importancia de $A E(\beta=0,34)$ es equivalente a la de los otros dos factores sumados. Si bien en la literatura la fuerza explicativa de los afectos en la intención de comportamiento ha sido a veces muy baja o no significativa (Bamberg \& Schmidt, 2003; Cheung et al., 2000), el hallazgo de este estudio se alinea con resultados que apoyan la importancia de este factor (Li \& Lai, 2008; Moody \& Siponen, 2013; Valois, Desharnais, \& Godin, 1988).

Finalmente, la proporción moderada de la varianza del comportamiento de estudio que es predicha por la intención y el hábito, especificada por el coeficiente de determinación $\left(R^{2}=0,54\right)$, nos señala que existen otros antecedentes o moderadores que pueden mejorar la explicación de esta variable. En ese sentido, las condiciones facilitadoras del estudio podrían jugar un rol importante, por ejemplo, si el estudiante universitario requiere balancear su tiempo de estudio 
con actividades laborales, su intención y hábito de estudio podrían ser moderadas por el tiempo que tiene para esa actividad.

\section{CONCLUSIÓN}

Esta investigación identificó un conjunto de factores que predicen la acción de estudiar por parte alumnos universitario chilenos. Si consideramos que el grado de motivación del estudiante universitario es decisivo para un aprendizaje efectivo, y el importante desafío en esta materia que enfrentan los sistemas de educación superior latinoamericanos y en particular el chileno, los hallazgos de este estudio incrementan el entendimiento de como este proceso de motivación se produce.

Desde el punto de vista práctico, los resultados del trabajo indican que un modelo basado en la TCl explica el comportamiento de estudio, y en particular, destacan al hábito de estudio como un antecedente clave de esta conducta. Consecuentemente, estos resultados implican que gestores y educadores universitarios podrían aumentar el grado de motivación de sus estudiantes a través del desarrollo mecanismos que posibiliten la formación de hábitos de estudio.

Desde el punto de vista teórico se deben destacar dos puntos. Primero, y si bien el hábito surge como una variable fundamental para explicar del estudio en alumnos universitarios, este trabajo también indica que este comportamiento se explica mejor si se considera además la intención consciente de estudiar. Y segundo, este estudio es el primero que ha validado un modelo $\mathrm{TCl}$ en un ámbito de educación superior latinoamericano, validación que posibilita su aplicación en otros contextos similares.

Ese estudio posee dos importantes limitaciones asociadas al trabajo empírico. Primero, dado las características de la muestra no es posible extrapolar en forma directa estos resultados. Y segundo, la encuesta fue aplicada en un solo momento del tiempo, lo que imposibilita evaluar como interactúan las variables hábito e intención en la explicación del comportamiento. Creemos que futuros trabajos deberían abordar estas limitaciones.

\section{REFERENCIAS BIBLIOGRÁFICAS}

1. AJZEN, I. (1991). The Theory of Planned Behavior. Organizational Behavior and Human Decision Processes, 50(2), 179-211.

2. AJZEN, I., \& FISHBEIN, M. (1980). Understanding attitudes and predicting social behaviour. Englewood Cliffs, NJ: Prentice-Hall.

3. AL-KHALDI, M. A., \& WALLACE, R. O. (1999). The influence of attitudes on personal computer utilization among knowledge workers: the case of Saudi Arabia. Information \& Management, 36(4), 185-204.

4. BAMBERG, S., \& SCHMIDT, P. (2003). Incentives, morality, or habit? Predicting students' car use for university routes with the models of Ajzen, Schwartz, and Triandis. Environment and behavior, 35(2), 264-285.

5. BEALANGER, D., GODIN, G., ALARY, M., \& BERNARD, P. M. (2002). Factors Explaining the Intention to Use Condoms Among Injecting Drug Users Participating in a Needle-Exchange Program. Journal of Applied Social Psychology, 32(5), 1047-1063. 
6. BOYD, B., \& WANDERSMAN, A. (1991). Predicting Undergraduate Condom Use with the Fishbein and Ajzen and the Triandis Attitude-Behavior Models: Implications for Public Health Interventions1. Journal of Applied Social Psychology, 21(22), 1810-1830.

7. BROWN, R. L., BAUMANN, L. J., HELBERG, C. P., HAN, Y., FONTANA, S. A., \& LOVE, R. R. (1996). The simultaneous analysis of patient, physician and group practice influences on annual mammography performance. Social science \& medicine, 43(3), 315-324.

8. CHEUNG, W., CHANG, M. K., \& LAI, V. S. (2000). Prediction of Internet and World Wide Web usage at work: a test of an extended Triandis model. Decision Support Systems, 30(1), 83-100.

9. CHIN, W. W. (1998). The partial least squares approach for structural equation modeling. In G. A. Marcoulides (Ed.), Modern Methods for Business Research (pp. 295-336). Hillsdale, NJ: Lawrence Erlbaum Associates.

10. DANNER, U. N., AARTS, H., \& VRIES, N. K. (2008). Habit vs. intention in the prediction of future behaviour: The role of frequency, context stability and mental accessibility of past behaviour. British Journal of Social Psychology, 47(2), 245-265.

11. DONOSO, S., \& SCHIEFELBEIN, E. (2007). Análisis de los modelos explicativos de retención de estudiantes en la universidad: una visión desde la desigualdad social. Estudios pedagógicos, 33(1), 7-27.

12. FERNÁNDEZ-HILEMAN, M. D. R., CORENGIA, Á., \& DURAND, J. (2014). Deserción y retención universitaria: una discusión bibliográfica. Pensando Psicología, 10(17), 85-96.

13. FORNELL, C., \& LARCKER, D. F. (1981). Evaluating Structural Equation Models with Unobservable Variables and Measurement Error. 18(1), 39-50.

14. KAPPE, R., \& VAN DER FLIER, H. (2012). Predicting academic success in higher education: what's more important than being smart? European Journal of Psychology of Education, 27(4), 605-619.

15. KARAHANNA, E., \& STRAUB, D. W. (1999). The psychological origins of perceived usefulness and ease-of-use. Information \& Management, 35(4), 237-250.

16. KENNEDY, G. J. (2013). The Elephant in the Hall: Motivating the Study of Student Motivation and Self-regulation in Studies of Academic Achievement and Persistence in Higher Education. International Journal of Higher Education, 2(4), 179-190.

17. KOCK, N. (2015). WarpPLS 5.0 user manual. Laredo, TX: ScriptWarp Systems.

18. LANKEN, B., AARTS, H., KNIPPENBERG, A., \& KNIPPENBERG, C. (1994). Attitude versus general habit: antecedents of travel mode Choice. Journal of Applied Social Psychology, 24(4), 285300.

19. LI, H., \& LAl, V. S. (2008). Antecedents of Behavioral Intention of Virtual Community Participation: An Empirical Study. AMCIS 2008 Proceedings, 326.

20. LIMAYEM, M., \& HIRT, S. G. (2003). Force of habit and information systems usage: theory and initial validation. Journal of the Association for Information Systems, 4(1), 65-97.

21. MATICKA-TYNDALE, E., HEROLD, E. S., \& MEWHINNEY, D. (1998). Casual sex on spring break: Intentions and behaviors of Canadian students. Journal of Sex Research, 35(3), 254-264.

22. MATICKA-TYNDALE, E., HEROLD, E. S., \& OPPERMANN, M. (2003). Casual sex among Australian schoolies. Journal of sex research, 40(2), 158-169. 
23. MOODY, G. D., \& SIPONEN, M. (2013). Using the theory of interpersonal behavior to explain non-work-related personal use of the Internet at work. Information \& Management, 50(6), 322-335.

24. ORTIZ, A. P., PÉREZ, V. R. D., \& SALAZAR, O. C. (2015). Una aproximación conceptual a la retención estudiantil en Latinoamérica. Revista Interamericana de Investigación, Educación y Pedagogía, 7(2), 271-285.

25. OUELLETTE, J. A., \& WOOD, W. (1998). Habit and intention in everyday life: the multiple processes by which past behavior predicts future behavior. Psychological bulletin, 124(1), 5474.

26. TENENHAUS, M., VINZI, V. E., CHATELIN, Y. M., \& LAURO, C. (2005). PLS path modeling. Computational Statistics \& Data Analysis, 48(1), 159-205.

27. TOUS, C., \& MEDINAS, M. (2007). Motivaciones para el estudio en universitarios. Anales de psicología, 23(1), 17-24.

28. TRIANDIS, H. (1980). Values, attitudes, and interpersonal behaviour. In H. E. Howe (Ed.), Nebraska symposium on motivation, 1979: Beliefs, attitudes and values (pp. 195-259). Lincoln, USA: University of Nebraska Press.

29. TRIANDIS, H. C. (1977). Interpersonal behavior. Monterey, CA: Brooks/Cole Publishing Company.

30. VALOIS, P., DESHARNAIS, R., \& GODIN, G. (1988). A comparison of the Fishbein and Ajzen and the Triandis attitudinal models for the prediction of exercise intention and behavior. Journal of Behavioral Medicine, 11(5), 459-472.

31. ZALESNY, M. D. (1985). Comparison of economic and noneconomic factors in predicting faculty vote preference in a union representation election. Journal of Applied Psychology, 70(2), 243. 